\title{
Electron subbands in a double quantum well in a quantizing magnetic field
}

\author{
V. T. Dolgopolov, A. A. Shashkin, and E. V. Deviatov \\ Institute of Solid State Physics, Chernogolovka, Moscow District 142432, Russia \\ F. Hastreiter, M. Hartung, and A. Wixforth \\ Ludwig-Maximilians-Universität, Geschwister-Scholl-Platz 1, D-80539 München, Germany \\ K. L. Campman and A. C. Gossard \\ Materials Department and Center for Quantized Electronic Structures, University of California, Santa Barbara, California 93106
}

(Received 9 June 1998; revised manuscript received 2 November 1998)

\begin{abstract}
We employ magnetocapacitance and far-infrared spectroscopy techniques to study the spectrum of the double-layer electron system in a parabolic quantum well with a narrow tunnel barrier in the center. For gate-bias-controlled asymmetric electron density distributions in this soft two-subband system we observe both individual subband gaps and double-layer gaps at integer filling factor $\nu$. The bilayer gaps are shown to be either trivially common for two subbands or caused by the wave function reconstruction in growth direction as induced by intersubband electron transfer at a normal to the interface magnetic field. In the latter case the observed gaps at $\nu=1$ and $\nu=2$ are described within a simple model for the modified bilayer spectrum. [S0163-1829(99)11319-5]
\end{abstract}

\section{INTRODUCTION}

The remarkable properties of a double electron layer in a quantizing magnetic field are determined not only by the relationship between the intralayer and interlayer Coulomb energies and the symmetric-antisymmetric level splitting caused by tunneling ${ }^{1-7}$ but also by the ratio of the distance between the subbands' electron density weight centers to the effective Bohr radius ${ }^{8}$

$$
\alpha=d / a_{B} .
$$

Throughout the paper we will bear in mind that the meaning of an individual layer is correct to introduce if the tunneling is small; otherwise, it only makes sense to speak about subbands with complicated electron density distributions in a double-layer system. So, the interlayer Coulomb correlations were shown to be responsible for the fractional quantum Hall effect at filling factor $\nu=1 / 2,{ }^{1-5}$ the many-body quantum Hall plateau at $\nu=1,{ }^{6,7}$ and the saturation of the phononinduced electron drag ${ }^{9}$ with lowering temperature. ${ }^{10}$ Besides, these were argued to destroy symmetric-antisymmetric splitting in strong magnetic fields. ${ }^{11-15}$

It is the ratio $\alpha$ that describes the softness of a bilayer electron system, i.e., the sensitivity of the subband spacing to intersubband electron transfer. ${ }^{16}$ Henceforth the symmetric double-layer regime where the electron density distribution is two symmetric maxima corresponding to two electron layers is referred to as balance. ${ }^{3-7}$ In view of softness, the balanced double layer, in which the subband electron density weight centers are coincident, is similar to a conventional two-subband electron system with vanishing $\alpha$, such as the one in single heterojunctions. Oppositely, the unbalanced double-layer system with asymmetric electron density distributions is normally soft, i.e., $\alpha \gtrsim 1$, and so its spectrum is very sensitive to intersubband charge transfer. In experiment, ${ }^{17}$ e.g., the Landau level fan chart peculiarities observed at relatively high filling factors in an unbalanced bilayer system were interpreted in terms of intersubband/ interlayer charge transfer without appealing exchange and correlation effects. Moreover, observation has been reported of hybrid gaps at filling factors $\nu=1,2$ at imbalance. ${ }^{16}$ The very different behaviors of the gap at $\nu=2$ at imbalance over the different ranges of bilayer electron densities have been detected and associated with a new phase transition. ${ }^{18} \mathrm{~A}$ broken-symmetry state in the fractional quantum Hall regime, whose formation is accompanied by intersubband/ interlayer charge transfer, has been observed in a wide quantum well. ${ }^{19}$ As was discussed in Ref. 20, the simplest effect of electron correlations is to change the partitioning of charge between two subbands/layers. It is interesting that the symmetry breaking does not necessarily imply nonzero charge transfer between subbands. At filling factor $\nu=2$, at balance, the electron correlations are expected to break the symmetry of the wave functions of electron subbands without causing intersubband charge transfer, which, in particular, points to the possible existence of a "canted antiferromagnetic" state. ${ }^{21}$

Here, using capacitive and far-infrared spectroscopy methods, we investigate the spectrum of two-dimensional electrons in a quantizing magnetic field in a parabolic quantum well that contains a narrow tunnel barrier for the electron systems on either side. Analysis of the behavior of the double-layer gaps at filling factors $\nu=1$ and $\nu=2$ at imbalance controlled by gate bias establishes the complete reconstruction of the zero-field wave functions in growth direction, caused by intersubband electron transfer in a magnetic field. A proposed model for the magnetic-field-induced reconstruction of electron subbands largely allows the description of the experimental results as well as recent data obtained in Ref. 18. 


\section{SAMPLES AND EXPERIMENTAL TECHNIQUE}

The samples are grown by molecular beam epitaxy on a semi-insulating GaAs substrate. The active layers form a 760 - $\AA$-wide parabolic well. In the center of the well a 3-monolayer-thick $\mathrm{Al}_{x} \mathrm{Ga}_{1-x} \mathrm{As}(x=0.3)$ sheet is grown which serves as a tunnel barrier between both parts on either side. The symmetrically doped well is capped by $600-\AA$ AlGaAs and $40-\AA$ GaAs layers. The samples have two Ohmic contacts (each of them is connected to both electron systems in two parts of the well) and two gates on the crystal surface with areas $120 \times 120$ and $220 \times 120 \mu \mathrm{m}^{2}$. The presence of the gate electrode enables us both to tune the carrier density in the well, which is equal to $4.2 \times 10^{11} \mathrm{~cm}^{-2}$ without gate bias, and measure the capacitance between the gate and the well. For capacitance measurements we apply an ac voltage $V_{\mathrm{ac}}=2.4 \mathrm{mV}$ at frequencies $f$ in the range $3-600 \mathrm{~Hz}$ between the well and the gate and measure both current components as a function of gate bias $V_{g}$, using a home-made $I-V$ converter and a standard lock-in technique. For farinfrared spectroscopy the sample design is a bit different: a 6 - $\mu \mathrm{m}$-period Ag grating coupler on the semitransparent gate is used to couple the normally incident far-infrared (FIR) radiation to the intersubband modes. Our measurements are performed in the temperature interval between $30 \mathrm{mK}$ and $2.5 \mathrm{~K}$ at magnetic fields of up to $16 \mathrm{~T}$.

The imaginary current component in the low-frequency limit reflects the thermodynamic density of states in a double-layer system in a quantizing magnetic field. This limit occurs if the sample dimension $L$ is small compared to the current overflowing length $L_{0}=\left(\sigma_{x x} / \pi f C\right)^{1 / 2}$, where $\sigma_{x x}$ is the dissipative conductivity of the bilayer system and $C$ is the capacitance per unit area between gate and quantum well. The imaginary current component minima are accompanied by peaks in the active current component which are proportional at low frequencies to $(f C)^{2} \sigma_{x x}^{-1}$ and can be used for measurements of $\sigma_{x x}$. In the high-frequency limit $L \gg L_{0}$ both components of the current tend to zero. Except for the balance point, our samples are similar to a conventional three-electrode system (see, e.g., Refs. 22 and 23), i.e., at imbalance the thermodynamic density of states is measured in the subband whose electron density maximum is closer to the gate. If the conductivity of the other subband does not vanish, the low-frequency limit is reached in the frequency range used. In this case one can expect weakly temperaturedependent relatively shallow minima in the sample capacitance that correspond to gaps in the spectrum of the former subband. Alternatively, if the Fermi level lies in a gap of the bilayer spectrum, at lowest temperatures the high-frequency limit is normally realized. This is indicated by deep minima in the imaginary current component that depend strongly on temperature. Hence, one can easily discriminate between one-subband gaps and gaps in the bilayer spectrum.

In principle, it would also be possible to make standard resistive measurements utilizing Corbino samples since this geometry allows one to avoid the interference of the Hall electric fields in two electron layers with different densities. Our arrangement takes advantage of the Corbino geometry and excels a standard technique in the simplicity of classifying the different kinds of minima.

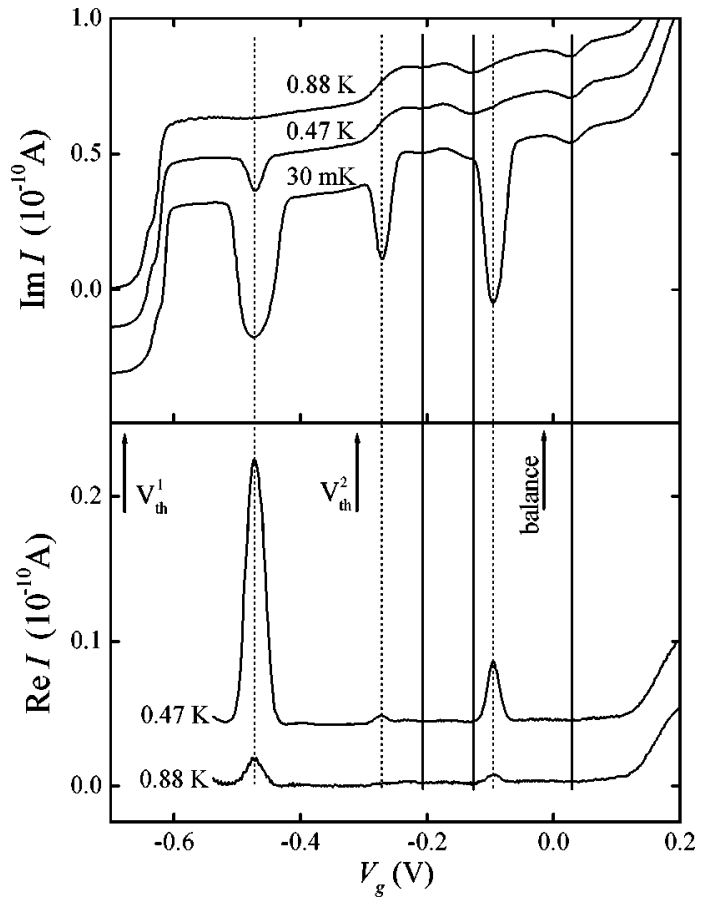

FIG. 1. Dependence of both current components on gate voltage at $f=300 \mathrm{~Hz}$ at different temperatures in a magnetic field of $2.5 \mathrm{~T}$. The lines for $30 \mathrm{mK}$ and $0.47 \mathrm{~K}$ are shifted for clarity. The vertical solid (dashed) lines mark minima of the density of states in the second subband (of the system conductivity). The positions of the subband thresholds and of the balance point are determined from the fan diagram in Fig. 2.

\section{RESULTS}

The dependences of both current components on gate voltage in a magnetic field of $2.5 \mathrm{~T}$ at different temperatures are represented in Fig. 1. At $V_{\text {th }}^{1}<V_{g}<V_{\text {th }}^{2}$ one subband is filled with electrons in the back part of the well, with respect to the gate. At the threshold voltage $V_{\text {th }}^{2}$ a second subband starts to collect electrons in the front part of the well, as indicated by a jump of the capacitance. Positions of the system conductivity minima are marked in the figure by dashed vertical lines. The solid lines mark minima of the thermodynamic density of states in the second electron subband. As seen from Fig. 1, the capacitance minima related to $\sigma_{x x}$ are strongly temperature dependent, whereas the measured capacitance in between the deep minima depends weakly on temperature. In the two-subband case the two kinds of minima coexist and, at close positions, switch with varying temperature because of very different temperature dependences. These determine two Landau level fans in the $\left(B, V_{g}\right)$ plane for the unbalanced double-layer system.

Figure 2 presents a Landau level fan chart for our sample. One-subband fans, i.e., those that correspond to individual electron subbands, are shown by dashed and dash-dotted lines, respectively. These are defined by minima of the thermodynamic density of states in the second subband at integer filling factor $\nu_{2}$ (here the experimental data points are not indicated to avoid overcomplicating the figure) and of the conductivity in the first subband at integer $\nu_{1}$. Their line slopes are inversely proportional to the capacitance values before and after the jump near $V_{g}=V_{\text {th }}^{2}$ (Fig. 1). The doublelayer conductivity minima at integer filling factor $\nu$ corre- 


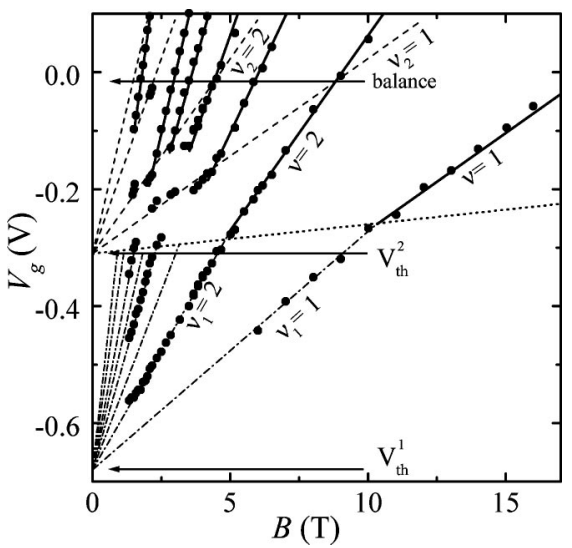

FIG. 2. Landau level fan chart as found from the minima of the density of states in the second electron subband at $\nu_{2}=1,2,4,6$ (dashed lines), of the conductivity in the first subband at $\nu_{1}$ $=1,2,4,6$ (dash-dotted lines), and of the double-layer conductivity at $\nu=1,2,3,4,5,6,8,10$ (solid lines). The change of fan line slopes occurs above the threshold $V_{\text {th }}^{2}$ as marked by dotted line.

sponding to gaps in the bilayer spectrum define the third, two-subband, Landau level fan as shown by solid lines in Fig. 2. These lines are parallel to the ones of the second subband fan so that with varying $V_{g}$ the bilayer electron density changes essentially in the front part of the well. The disruptions of the two-subband fan lines at $\nu>2$ imply the disappearance of common gaps for two subbands as will be discussed below. As seen from Fig. 2, the two-subband and first-subband fan lines for each $\nu=\nu_{1}$ intercept one another near the dotted line at $V_{g}>V_{\text {th }}^{2}$. For description of all of these fan lines we will use filling factor $\nu$ since $\nu_{1}$ at $V_{g}$ $<V_{\text {th }}^{2}$ is also determined by the electron density $N_{s}$ in the quantum well. A part of the fan chart including the data points for the thermodynamic density of states minima in the second subband is displayed in Fig. 3. At low magnetic fields the measurements are taken at the lowest temperatures to obtain more pronounced capacitance minima while at high $B$ the temperatures of about $1 \mathrm{~K}$ are necessary to suppress the bilayer conductivity minima. These limits are indicated by different symbols in the figure. The small deviations of the data from the dashed lines give evidence that the intersubband/interlayer electron transfer is small and the unbalanced double-layer system is soft.

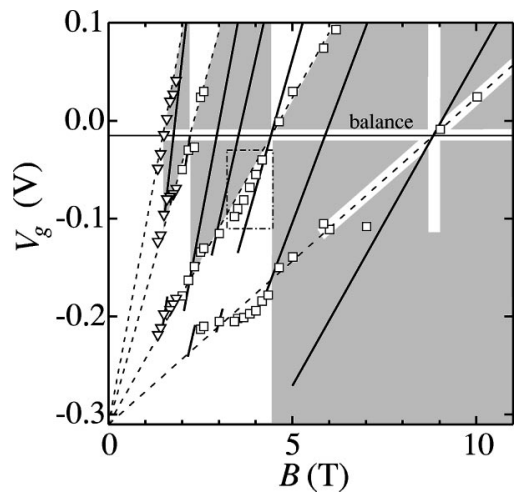

FIG. 3. Part of the fan chart in Fig. 2, including the data points for density of states minima in the second subband. The reconstruction of electron subbands is expected in shaded regions.

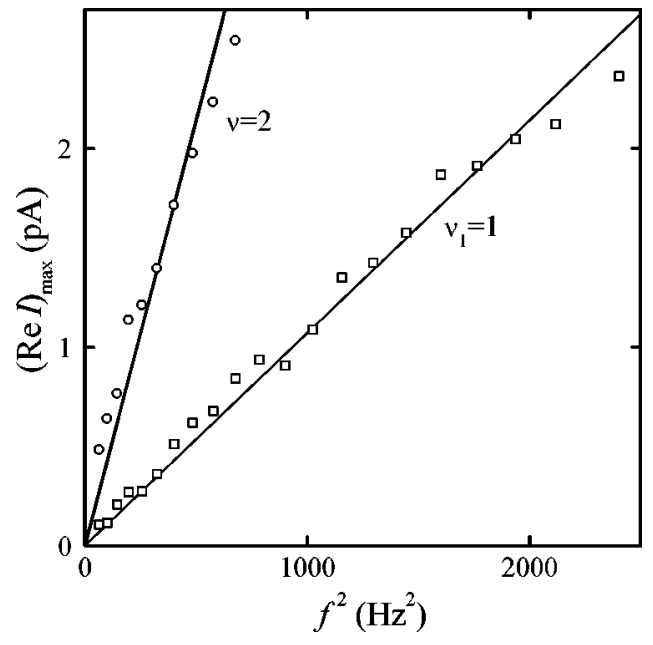

FIG. 4. Frequency dependence of the active current component peaks at $B=8 \mathrm{~T}$ at $T=0.62 \mathrm{~K}$.

Activation energy for gaps at integer $\nu$ is found from the temperature dependence of peaks in the active current component. We have checked that at sufficiently low frequencies the peak amplitude is a parabolic function of $f$, see Fig. 4. As mentioned above, the slopes of the straight lines in the figure should be inversely proportional to $\sigma_{x x}$, which justifies the procedure of activation energy determination from the Arrhenius plot of the peak amplitude.

A typical behavior of the activation energy $E_{a}$ with magnetic field at fixed filling factor $\nu>2$ is depicted in Fig. 5 for the case of $\nu=4$. The value of $E_{a}$ is a maximum both at the bilayer onset $V_{\text {th }}^{2}$ and at balance. In between these it goes to zero in the interval 2.6-3.4 $\mathrm{T}$ except for the close vicinity of $3 \mathrm{~T}$ where $E_{a}$ is unmeasurably small but likely finite as can be reconciled with the nonzero active current component arising at a fan crossing point $\nu=4, \nu_{2}=1$ (cf. Figs. 2, 5). The disruptions of the two-subband fan lines are the case between Landau level fan crossings (Fig. 2) and correspond to the intervals of $B$ (or $V_{g}$ ) in which the activation energy vanishes (Fig. 5).

In contrast, at $\nu=1,2$ the activation energy in the bilayer system never tends to zero (Figs. 6, 7). For both filling fac-

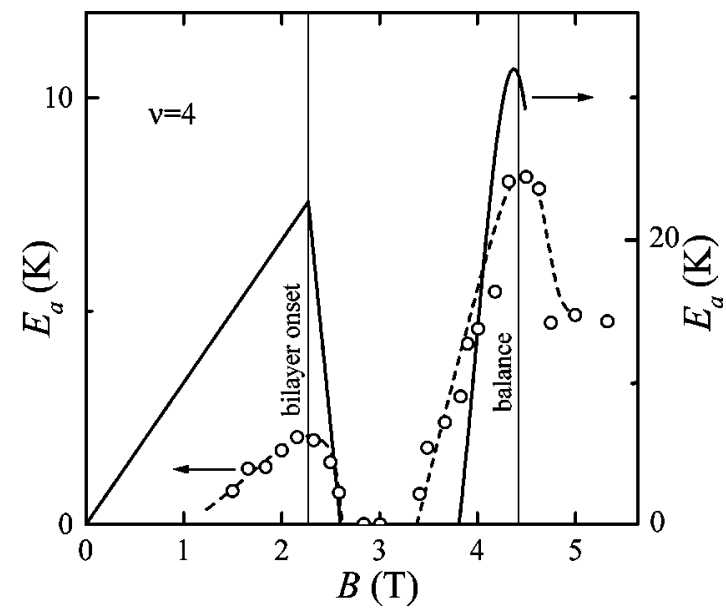

FIG. 5. Experimental (dots) and calculated (solid line) changes of the activation energy with magnetic field for filling factor $\nu=4$. The dashed line is a guide to the eye. 

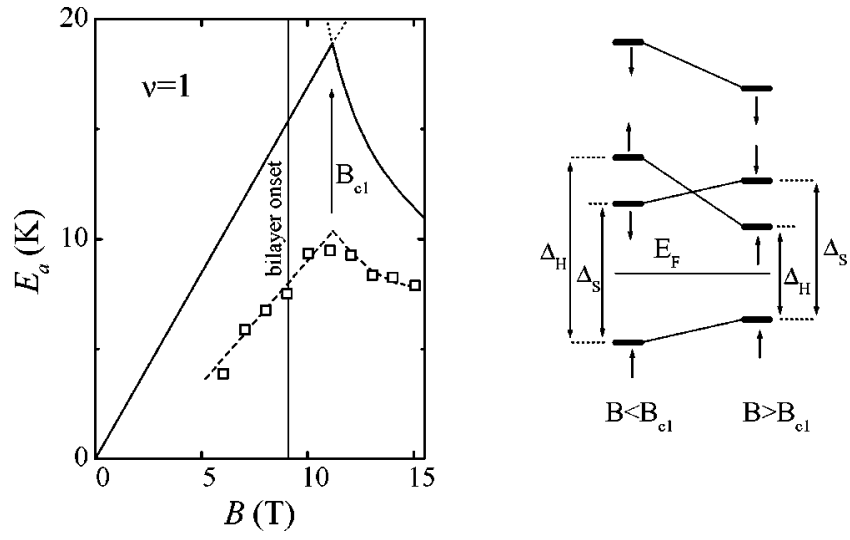

FIG. 6. Activation energy as a function of magnetic field at $\nu$ $=1$ : experiment (squares) and calculation (solid line). The dashed line is a guide to the eye. Also shown is a sketch of the bilayer spectrum below and above the maximum field $B_{c 1}$.

tors $E_{a}$ is a maximum near $V_{g}=V_{\text {th }}^{2}$ and then it monotonically decreases with magnetic field up to the balance point. We note that these $E_{a}$ maxima are attained at the interception points of the two-subband and first-subband fan lines as described above (Fig. 2).

Figure 8 shows FIR absorption spectra in magnetic field at $V_{g}=-0.15 \mathrm{~V}$ on our sample. Line $\mathrm{C}$ is easy to identify as the cyclotron resonance owing to the proportional $B$ dependence of its energy with the slope as determined by an effective mass $0.07 m_{0}$ ( $m_{0}$ is the free electron mass). The cyclotron resonance is observed with or without grating coupler on the top of the sample. According to Ref. 24, the other three lines reflect the single-electron spectrum in the quantum well, taking account of depolarization shift. All of these are seen only in the presence of a grating coupler, which is in agreement with their intersubband origin. Two lines $A$ and $B$ are attributed to electron transitions between the second and fourth subband and the first and third subband, respectively. ${ }^{24}$ The most interesting line $D$ emerges at a magnetic field of $6 \mathrm{~T}$ corresponding to $\nu \approx 2$ and its energy is nearly independent of $B$ (Fig. 8). This is the $D$ line which we attribute to a hybrid gap in the double-layer spectrum.
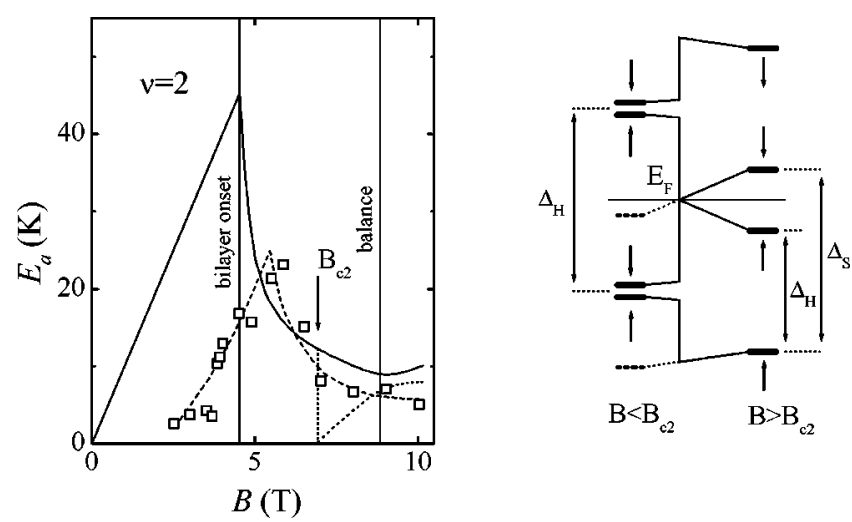

FIG. 7. Comparison of the experimental (squares) and theoretical (solid and dotted lines) dependences of the activation energy on magnetic field for $\nu=2$. The dashed line is a guide to the eye. The sketch displays the expected bilayer spectrum below and above the critical field $B_{c 2}$.
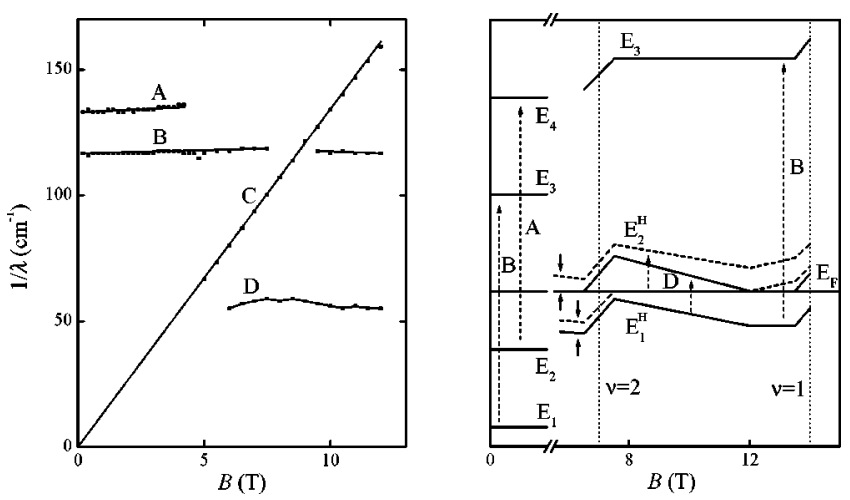

FIG. 8. FIR absorption spectra at different magnetic fields at $V_{g}=-0.15 \mathrm{~V}$. Also shown is the schematic energy spectrum with the corresponding transitions in magnetic field.

\section{DISCUSSION}

The band structure of our sample in the absence of magnetic field is known from far-infrared spectroscopy and magnetotransport investigations on samples fabricated from the same wafer. ${ }^{25,26}$ It agrees with the result of self-consistent Hartree calculation of energy levels in a coupled double quantum well. According to the calculation, the symmetricantisymmetric splitting in the balanced bilayer system, which is the case at zero gate voltage, is equal to $\Delta_{\mathrm{SAS}}=1.3 \mathrm{meV}$. With decreasing $V_{g}$ the difference in the second- and firstsubband energies should enhance achieving the value of 6.7 $\mathrm{meV}$ at the bilayer onset. The calculated electron density profiles $\left|\psi_{1,2}\right|^{2}$ for both subbands at two gate voltages are displayed in the insets to Fig. 9. Because of tunneling the wave functions are not localized in either part of the quantum well. The corresponding electron density distributions in the quantum well $\rho=N_{s}^{1}\left|\psi_{1}\right|^{2}+N_{s}^{2}\left|\psi_{2}\right|^{2}$ (where $N_{s}^{1,2}$ are the subbands' electron densities) are shown in Fig. 9 by dashed lines.

For an unbalanced double layer, switching a quantizing magnetic field results for the simplest case in intersubband/ interlayer electron transfer $n_{s}$ to minimize the system energy that is accompanied by a relative shift of Landau level ladders for two subbands. This shift can be estimated at

$$
\Delta \approx 4 \pi e^{2} n_{s} d / \varepsilon
$$

The system softness $\alpha$ is characterized by the ratio of the shift $\Delta / 2$ of an individual ladder caused by transfer of all electrons in the Landau level to the cyclotron energy, which is consistent with the relation (1). Given two Landau level ladders that correspond to one-subband fans, the origin of the third two-subband Landau level fan is explained in the following way. At fixed integer $\nu$ the Fermi level $E_{F}$ can be expected either to pin to both quantum levels for two subbands or fall within a common gap of the spectrum. The latter should occur around the fan crossing points, at which both $\nu_{1}$ and $\nu_{2}$ are integer, whereas in the pinning regions in between the common gap should close. It is easy to obtain that the ratio of the region dimensions where the common gap is absent and, respectively, present is proportional to $\alpha$. Hence, in a conventional two-subband system with vanishing $\alpha$ the pinning regions should reduce to points and, oppositely, in a soft two-subband system with $\alpha \gtrsim 1$ these should 


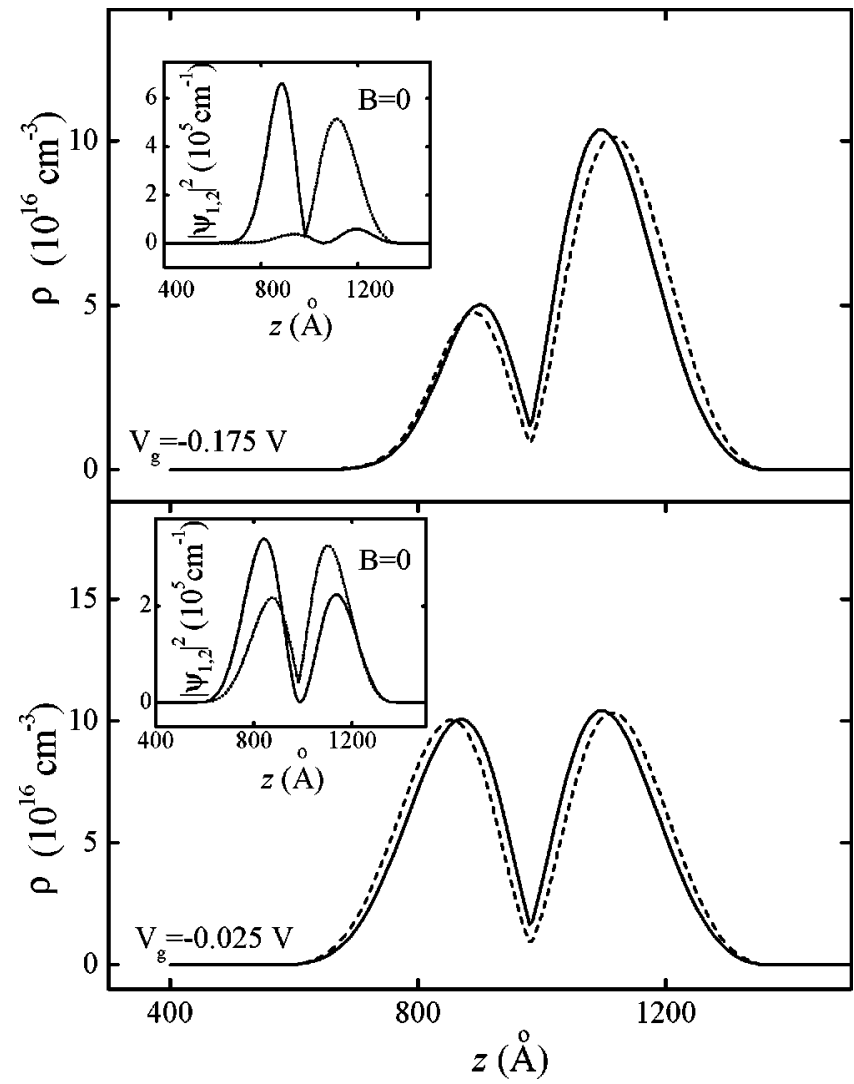

FIG. 9. Calculated electron density distributions in the quantum well for two gate voltages at zero magnetic field (dashed lines) and at filling factor $\nu=1$ (solid lines). The $z$ coordinate is counted from the crystal surface. The electron density profiles for both subbands at $B=0$ are shown in the insets.

extend up to fan crossing points. The latter statement is in agreement with the data at $\nu>2$ for our double layer in which $\alpha$ reaches 2.5, see Figs. 2 and 9. However, this crude approach which deals with zero-magnetic-field subbands in an unbalanced double layer is not good at filling factor $\nu$ $=1,2$, at least (Fig. 2).

To obtain more rigorous solution of the problem in magnetic field we start from the zero $B$ solution and determine the first order corrections $\Delta_{i j}$ to the subband energies $E_{1}$ and $E_{2}$, caused by intersubband charge transfer in a magnetic field

$$
\begin{aligned}
\Delta_{i j}= & \frac{4 \pi e^{2}}{\varepsilon} \int_{-\infty}^{\infty} \Theta_{i k}^{*}(x, y) \Theta_{j l}(x, y) d x d y \psi_{i}^{*}(z) \psi_{j}(z) d z \\
& \times \int_{-\infty}^{z} d z^{\prime} \int_{-\infty}^{z^{\prime}} \Delta \rho\left(z^{\prime \prime}\right) d z^{\prime \prime}, \quad i, j=1,2 .
\end{aligned}
$$

Here $\Theta_{i k}$ is the wave function of the level with Landau quantum number $k$ in the $i$ th subband, which is closest to the Fermi level, and $\Delta \rho$ is the variation of the electron density distribution when switching the magnetic field. In our simple model we will disregard exchange and correlation effects.

In the region of common gaps for two subbands (unshaded area in Fig. 3) the quantum level numbers $k, l$ for $i$ $\neq j$ are different and so because of the orthogonality of $\Theta_{i k}$ and $\Theta_{j l}$ the off-diagonal $\Delta_{i j}$ are equal to zero, leaving the wave functions $\psi_{1,2}$ unchanged. This region is confined by the second subband fan lines and the expected vertical fan lines for the first subband as shown in the figure. The value $\Delta \rho$ here is given by

$$
\Delta \rho=\left(\left|\psi_{2}\right|^{2}-\left|\psi_{1}\right|^{2}\right) n_{s},
$$

where $n_{s}$ is electron transfer from the second to the first subband. One can see from Eqs. (3) and (4) that in the general case the sum $\Delta_{11}+\Delta_{22} \neq 0$. At integer $\nu$ the intersubband/interlayer electron transfer gives rise to one of the following: either both of the quantum levels in question reach the Fermi level so that the common gap collapses or the top quantum level becomes empty, keeping a gap. That is, $n_{s}$ is determined from the condition of the common gap collapse if the top quantum level remains filled after electron transfer and is equal to the electron density in the top quantum level otherwise. Obviously, the latter occurs near the fan crossing points. In the simplest case of equal gaps for two subbands the common gap reduces by $\left|\Delta_{22}-\Delta_{11}\right|$.

The result of the common gap calculation at $\nu=4$ using the above procedure is shown in Fig. 5. To allow for the discrepancy between the calculated and measured values of sample capacitance, the $B$ values for the theoretical dependence $E_{a}(B)$ are multiplied by a factor of 1.1 to fit the known electron density. For the sake of simplicity the spin splitting is ignored and so there are only two maxima on the expected dependence of the activation energy on magnetic field. Although the qualitative agreement with the experiment is good, the expected values of $E_{a}$ are very different from the experimental data. This is likely to be due to a disorder in the double layer that leads to the finite width of quantum levels which is neglected in the calculations.

We note that, if the Fermi level lies in a common gap, the intersubband/interlayer charge transfer is given by deviations of the experimental data for the second-subband fan from the straight lines (see, e.g., the region marked by a rectangle in Fig. 3). That these deviations are small excludes the possibility of all electrons collecting in one part of the quantum well (so-called broken-symmetry states ${ }^{20}$ ).

The above calculations are straightforward and analogous to numerical calculations made in previous publications, e.g., in Ref. 17. Below we will show that, if the orthogonality of $\Theta_{i k}$ and $\Theta_{j l}$ does not hold, $\psi_{1,2}$ at imbalance are not eigenfunctions of the problem with the perturbation (3) any longer. Mixing the states $\psi_{1,2}$ is equivalent with intersubband but not interlayer electron transfer at a magnetic field and naturally leads to the appearance of new reconstructed subbands that are similar to the zero $B$ subbands at balance. As a result, there emerges a hybrid gap that does not collapse at integer $\nu$ in the shaded region of Fig. 3. We call this gap hybrid to emphasize its relation to the magnetic-fielddependent wave function reconstruction in the $z$ direction.

Over shaded areas in Fig. 3, the Landau level numbers $k$ and $l$ for $i \neq j$ in Eq. (3) are coincident so that the offdiagonal perturbation terms $\Delta_{i j} \neq 0$ and the solution should be searched in the form of linear combination of $\psi_{1,2}$. To simplify the problem, we will not mix states with antiparallel spins, ignoring spin flip processes in tunneling. This is reasonable once the exchange and correlation effects are ne- 
glected. Then, in the Hartree approximation one has to solve the determinant equation (for certainty we consider the case of $k=l=0$ )

$$
\operatorname{det}\left(\begin{array}{cc}
\Delta_{11}+E_{1}-E_{1,2}^{H} & \Delta_{12} \\
\Delta_{21} & \Delta_{22}+E_{2}-E_{1,2}^{H}
\end{array}\right)=0
$$

to find the reconstructed subband energies $E_{1,2}^{H}$. These states are described by the mixed wave functions

$$
\begin{aligned}
\psi_{1,2}^{H}= & {\left[\Delta_{12}^{2}+\left(\Delta_{11}+E_{1}-E_{1,2}^{H}\right)^{2}\right]^{-1 / 2} } \\
& \times\left[\Delta_{12} \psi_{1}-\left(\Delta_{11}+E_{1}-E_{1,2}^{H}\right) \psi_{2}\right]
\end{aligned}
$$

and thus instead of Eq. (4) we get for $\Delta \rho$ the following expression at $\nu=1$ :

$$
\Delta \rho=\left(N_{s}^{1}+N_{s}^{2}\right)\left|\psi_{1}^{H}\right|^{2}-N_{s}^{1}\left|\psi_{1}\right|^{2}-N_{s}^{2}\left|\psi_{2}\right|^{2},
$$

where $\psi_{1}^{H}$ corresponds to the lower-energy state $E_{1}^{H}$. Equations (3) and (5) - (7) compose a perturbation theory loop for self-consistent solution of the problem. We solve it for the case of spinless electrons at $\nu=1$, although the procedure holds for any $\nu$ in the shaded region of Fig. 3. As compared to a common gap for two subbands, the hybrid gap $\Delta_{H}$ $=E_{2}^{H}-E_{1}^{H}<E_{2}-E_{1}$ never goes to zero since it determines energy spacing between the subbands that is expected at minimum to be equal to $\Delta_{\mathrm{SAS}}$. So, the magnetic-fieldinduced wave function reconstruction in the $z$ direction generalizes the case of symmetric electron density distributions corresponding to formation of $\Delta_{\text {SAS }}$.

It is interesting to compare how the zero-magnetic-field electron density distribution in the quantum well changes for common and hybrid gaps. From Eq. (4) it follows that in the case of a common gap some charge is transferred between the front and back parts of the well, i.e., $\Delta \rho(z)$ is close to an antisymmetric function and so the corrections to the subband energies $E_{1}, E_{2}$ are of opposite sign. In contrast, for a hybrid gap electrons are displaced nearly symmetrically toward the well center (Fig. 9), resulting in the energy shifts of the same $\operatorname{sign} E_{1}^{H}>E_{1}$ and $E_{2}^{H}>E_{2}$.

Now in our model we introduce the spin splitting. Since the expected hybrid splitting $\Delta_{H}$ is comparable to the manybody enhanced spin splitting $\Delta_{S}$, the energy spectrum should be determined by their competition. For the simplest case of $\nu=1$ the many-body enhanced spin gap as estimated for the effective $g$ factor of 5.2 (Ref. 23) is dominant over the range of magnetic fields used except near the bilayer onset where the hybrid splitting approaches half of the cyclotron energy. That stands to reason; it is the smaller splitting that is the place at filling factor $\nu=1$. Therefore, the theoretical dependence is a maximum at a magnetic field $B_{c 1}$ above the second-subband threshold. This is in reasonable agreement with the experiment (see Figs. 6, 2) as the actual value of effective $g$ factor may be smaller because of disorder.

For filling factor $\nu=2$ the situation is far more sophisticated because, first, the actual gap is given by the splittings' difference and, secondly, the $g$ factor can be enhanced or not, dependent on filling of the spin sublevels. At least, near the bilayer onset the hybrid gap exceeds the estimated manybody enhanced spin gap. As long as in this case the spin sublevels with antiparallel spins are filled, the spin splitting should correspond to the $g$ factor in bulk GaAs, $|g|=0.44$. This is true until the hybrid and many-body enhanced spin splittings become equal at a higher magnetic field $B_{c 2}$ (Fig. 7 ). If $B>B_{c 2}$, it is energetically more favorable for the system to have the spin splitting enhanced so that the spin sublevels with parallel spins are filled. Hence, the expected gap at $\nu=2$ should collapse around $B=B_{c 2}$ and then increase with magnetic field as shown in the figure. Yet, this scenario is not realized in our double layer. This implies that the actual many-body enhanced $g$ factor is smaller than the one used in the calculation, i.e., the hybrid gap is dominant over the entire range of fields up to the balance point. Therefore, in the other scenario the gap at $\nu=2$ should merely be equal to the difference between the hybrid and Zeeman splittings, which is in qualitative agreement with the data (Fig. 7). Thus, in our experiment both gaps at $\nu=1,2$ are of hybrid origin as caused by the interplay of the hybrid and spin splittings. Apparently, for odd $\nu>1$ near the balance one can expect gaps of spin origin that are the smallest at lower magnetic fields. We note that the above scenarios completely account for results of activation energy measurements in a double-layer system at filling factor $\nu=2$ of Ref. 18: at low electron densities in a double layer the behavior of $E_{a}$ at $\nu$ $=2$ found in Ref. 18 is similar to ours, whereas at higher electron densities, i.e., higher magnetic fields and larger spin gaps, $E_{a}$ has been observed to collapse near the balance point, as expected from the first scenario.

With respect to the FIR data, we emphasize that, since the wavelength of FIR excitation is large compared to the distance between electrons, the FIR absorption spectra should be of collective origin. Indeed, the cyclotron resonance line $C$, for instance, reflects the coherent behavior of twodimensional electrons during transition, which is consistent with Kohn's theorem. On the other hand, it was shown theoretically that the measured splitting in the collective intersubband spectra does reveal the single-particle splitting ${ }^{27,28}$ and so for this case the single-electron picture can be used.

In the absence of magnetic field, at balance, four intersubband transitions $\quad E_{1} \rightarrow E_{3}, \quad E_{1} \rightarrow E_{4}, \quad E_{2} \rightarrow E_{3}, \quad E_{2}$ $\rightarrow E_{4}$ were detected in FIR studies on a sample with the same structure, ${ }^{24}$ while at imbalance we observe only two transitions $A$ and $B$ (Fig. 8). Since at balance all wave functions are symmetric in the $z$ direction, the suppression of the other two transitions is likely to be caused by the different symmetry of the subband wave functions with odd and even numbers at imbalance. In a magnetic field the wave function symmetry changes because of mixing of the subbands $E_{1}$ and $E_{2}$ to form the reconstructed subbands $E_{1}^{H}$ and $E_{2}^{H}$, which is accompanied by the second-subband depopulation (Fig. 8). As a result, one can expect that with increasing $B$, near the reconstruction region boundary, the $A$ line intensity should drop and a new line $D$ should emerge. From comparison of Figs. 3, 8 it follows that switching these lines indeed happens near the shaded area boundary at $B=4.4 \mathrm{~T}$. This as well as the weak magnetic field dependence of the energy of line $D$ confirm its hybrid origin. As mentioned above, the depolarization shift describing collective effects in the single-electron spectrum in the quantum well should be taken into account to extract the subband energies from the data obtained with zero wave-vector excitation. ${ }^{24}$ That the depolarization shift is difficult to compute encumbers the 
comparison of the $D$ line energy with the calculated hybrid splitting; nevertheless, this shift is not expected to depend strongly on magnetic field.

Although adequate, our approach does not explain satisfactorily the presence of data points at $\nu_{2}=1$ in the reconstruction region (Fig. 3). In principle, peculiarities are indeed expected in the vicinity of one-subband fan lines for odd $\nu_{1}$ and $\nu_{2}$ since the intersubband electron transfer cannot occur there if $E_{2}-E_{1}<\Delta_{S}$. This inequality determines the regions where the subbands should be original (see the white strips in Fig. 3, including the balance region). In fact, for our case the above inequality is never fulfilled, as follows from the hybrid origin of the gap at $\nu=2$ (cf. Figs. 3, 7). This contradiction is not resolved within the simple model above and demands consideration of exchange and correlation effects.

\section{CONCLUSION}

In summary, we have performed magnetocapacitance and FIR measurements on a bilayer electron system in a parabolic quantum well with a narrow tunnel barrier in its center. At imbalance created by gate depletion we observe individual subband gaps and gaps in the double-layer spectrum at integer $\nu$. The latter are analyzed to be either common for two subbands or caused by the wave function reconstruction in growth direction at a magnetic field. With the help of a model for the magnetic-field-induced reconstruction of electron subbands, we explain the observed behavior of the double-layer gaps at $\nu=1$ and $\nu=2$ as well as data of Ref. 18 on collapse of the gap at $\nu=2$ near the balance point, making allowance for the competition between the hybrid and spin splittings.

\section{ACKNOWLEDGMENTS}

We gratefully acknowledge J.P. Kotthaus, A.V. Chaplik, and M. Shayegan for fruitful discussions of the results. This work was supported in part by the Deutsche Forschungsgemeinschaft, the AFOSR under Grant No. F49620-94-1-0158, the Russian Foundation for Basic Research under Grants No. 97-02-16829 and No. 98-02-16632, and the program "Nanostructures" from the Russian Ministry of Sciences under Grant No. 97-1024. The Munich-Santa Barbara collaboration has also been supported by a joint NSF-European Grant and the Max-Planck research award.
${ }^{1}$ T. Chakraborty and P. Pietilainen, Phys. Rev. Lett. 59, 2784 (1987).

${ }^{2}$ D. Yoshioka, A. H. MacDonald, and S. M. Girvin, Phys. Rev. B 39, 1932 (1989).

${ }^{3}$ J. P. Eisenstein, G. S. Boebinger, L. N. Pfeiffer, K. W. West, and S. He, Phys. Rev. Lett. 68, 1383 (1992).

${ }^{4}$ Y. W. Suen, L. W. Engel, M. B. Santos, M. Shayegan, and D. C. Tsui, Phys. Rev. Lett. 68, 1379 (1992).

${ }^{5}$ Y. W. Suen, H. C. Manoharan, X. Ying, M. B. Santos, and M. Shayegan, Phys. Rev. Lett. 72, 3405 (1994).

${ }^{6}$ S. Q. Murphy, J. P. Eisenstein, G. S. Boebinger, L. N. Pfeiffer, and K. W. West, Phys. Rev. Lett. 72, 728 (1994).

${ }^{7}$ T. S. Lay, Y. W. Suen, H. C. Manoharan, X. Ying, M. B. Santos, and M. Shayegan, Phys. Rev. B 50, 17725 (1994).

${ }^{8}$ A. Gold, Z. Phys. B 95, 341 (1994).

${ }^{9}$ T. J. Gramila, J. P. Eisenstein, A. H. MacDonald, L. N. Pfeiffer, and K. W. West, Phys. Rev. Lett. 66, 1216 (1991).

${ }^{10}$ M. P. Lilly, J. P. Eisenstein, L. N. Pfeiffer, and K. W. West, Phys. Rev. Lett. 80, 1714 (1998).

${ }^{11}$ G. S. Boebinger, H. W. Jiang, L. N. Pfeiffer, and K. W. West, Phys. Rev. Lett. 64, 1793 (1990).

${ }^{12}$ Y. W. Suen, J. Jo, M. B. Santos, L. W. Engel, S. W. Hwang, and M. Shayegan, Phys. Rev. B 44, 5947 (1991).

${ }^{13}$ A. H. MacDonald, P. M. Platzman, and G. S. Boebinger, Phys. Rev. Lett. 65, 775 (1990).

${ }^{14}$ L. Brey, Phys. Rev. Lett. 65, 903 (1990).

${ }^{15}$ S. He, X. C. Xie, S. Das Sarma, and F. C. Zhang, Phys. Rev. B 43, 9339 (1991).

${ }^{16}$ V. T. Dolgopolov, G. E. Tsydynzhapov, A. A. Shashkin, E. V. Deviatov, F. Hastreiter, M. Hartung, A. Wixforth, K. L. Camp- man, and A. C. Gossard, Pis'ma Zh. Éksp. Teor. Fiz. 67, 563 (1998) [JETP Lett. 67, 595 (1998)].

${ }^{17}$ A. G. Davies, C. H. W. Barnes, K. R. Zolleis, J. T. Nicholls, M. Y. Simmons, and D. A. Ritchie, Phys. Rev. B 54, R17 331 (1996).

${ }^{18}$ A. Sawada, Z. F. Ezawa, H. Ohno, Y. Horikoshi, Y. Ohno, S. Kishimoto, F. Matsukara, M. Yasumoto, and A. Urayama, Phys. Rev. Lett. 80, 4539 (1998).

${ }^{19}$ H. C. Manoharan, Y. W. Suen, T. C. Lay, M. B. Santos, and M. Shayegan, Phys. Rev. Lett. 79, 2722 (1997).

${ }^{20}$ T. Jungwirth and A. H. MacDonald, Phys. Rev. B 53, 9943 (1996).

${ }^{21}$ L. Zheng, S. Sachdev, and S. Das Sarma, Phys. Rev. B 58, 4672 (1998).

${ }^{22}$ R. C. Ashoori and R. H. Silsbee, Solid State Commun. 81, 821 (1992).

${ }^{23}$ V. T. Dolgopolov, A. A. Shashkin, A. V. Aristov, D. Schmerek, H. Drexler, W. Hansen, J. P. Kotthaus, and M. Holland, Phys. Low-Dimens. Semicond. Struct. 6, 1 (1996); V. T. Dolgopolov, A. A. Shashkin, A. V. Aristov, D. Schmerek, W. Hansen, J. P. Kotthaus, and M. Holland, Phys. Rev. Lett. 79, 729 (1997).

${ }^{24}$ M. Hartung, A. Wixforth, K. L. Campman, and A. C. Gossard, Superlattices Microstruct. 19, 55 (1996).

${ }^{25}$ M. Hartung, A. Wixforth, K. L. Campman, and A. C. Gossard, Solid-State Electron. 40, 113 (1996).

${ }^{26}$ G. Salis, B. Graf, K. Ensslin, K. Campman, K. Maranowski, and A. C. Gossard, Phys. Rev. Lett. 79, 5106 (1997).

${ }^{27}$ K. Leo, J. Shah, J. P. Gordon, T. C. Damen, D. A. B. Miller, C. W. Tu, and J. E. Cunningham, Phys. Rev. B 42, 7065 (1990).

${ }^{28}$ M. Zaluzny, Appl. Phys. Lett. 65, 1817 (1994). 